\title{
Voltage-Clamp Analysis of Sodium Channels in Wild-type and Mutant Drosophila Neurons
}

\author{
Diane K. O'Dowd and Richard W. Aldrich \\ Department of Neurobiology, Stanford University School of Medicine, Stanford, California 94305
}

In this study we describe a preparation in which we examined directly, using tight-seal whole-cell recording, sodium currents from embryonic Drosophila neurons maintained in culture. Sodium currents were expressed in approximately $65 \%$ of the neurons prepared from wild-type Drosophila embryos when examined at room temperature, $24 \mathrm{hr}$ after plating. While current density was low, other features of the sodium current in wild-type neurons, including the voltage sensitivity, steady-state inactivation, macroscopic time course, and TTX sensitivity were similar to those found in other excitable cells. Physiological and biochemical evidence has led to the suggestion that mutations in the nap, seizure, and tip-E loci of Drosophila may affect voltage-dependent sodium channels. There was no significant difference in the percentage of neurons expressing sodium currents in cultures prepared from embryos with mutations at the nap, sei or tip-E loci compared with wild-type cultures. Sodium currents recorded from napts appeared similar in all of the properties examined to those in wild-type cells. However, neuronal sodium current density was $40-60 \%$ lower in cultures prepared from both tip- $E$ and se ${ }^{i s t}$ embryos. The voltage dependence and gating properties of these sodium channels, as well as the TTX sensitivity, appear similar to wild type. These results indicate that both the tip- $E$ and sei loci are important in regulation of sodium current density in embryonic neurons.

The importance of voltage-gated sodium channels in Drosophila neurons has been inferred from electrophysiological studies documenting TTX-sensitive spikes in the axons of the giant fiber system. In addition, biochemical studies have revealed the presence of high-affinity sites for ligands that bind to voltage-gated sodium channels, TTX and saxitoxin (STX), in membranes prepared from adult fly heads. Recently, 2 regions of the Drosophila genome have been identified that have high sequence similarity with vertebrate genes that code for alpha subunits of sodium channels (Salkoff et al., 1987; K. Loughney and B. Ganetzky, personal communication). The potential for combined molecular, genetic, and physiological studies makes Drosophila a valuable system for the study of the function and regulation

Received Aug. 17, 1987; revised Feb. 19, 1988; accepted Feb. 22, 1988.

We thank Dr. Susan Germeraad for helpful discussions and assistance throughout this project and Dr. L. Byerly for advice on embryo tissue culture techniques. We also thank Drs. M. A. Smith. T. Hoshi, and D. Baylor for comments on the manuscript. This work was supported by an American Cancer Society postdoctoral fellowship to D.K.O.D. and NIH NS23294 and a fellowship from the Searle Scholars Program/Chicago Community Trust to R.W.A.

Correspondence should be addressed to Diane K. O'Dowd at the above address. Copyright (C) 1988 Society for Neuroscience $0270-6474 / 88 / 103633-11 \$ 02.00 / 0$ of neuronal sodium channels. However, direct physiological examination of sodium currents in adult and larval Drosophila neurons has proven difficult due to the small size and relative inaccessibility of the neurons. With the goal of identifying regions of the Drosophila genome important in the functional expression of neuronal sodium channels, including both genes coding for structural components of the channels and those involved in their regulation, we have utilized a preparation in which we could biophysically examine isolated neuronal sodium currents (Seecof, 1979; Byerly, 1985). In this article we describe conditions in which voltage-clamped sodium currents can be studied in cultured Drosophila neurons from both wild-type and mutant embryos.

Behavioral mutations have been used to identify a number of genes involved in the generation of electrical excitability in Drosophila nerve and muscle cells (see reviews by Hall, 1982; Ganetzky and $\mathrm{Wu}, 1986$; Salkoff and Tanouye, 1986; Tanouye et al., 1986). Some of these mutants-including $n a p^{t s}$, $s e i^{i s}$, and tip-E-were originally isolated on the basis of a rapid and reversible temperature-sensitive paralytic phenotype (Wu et al., 1978; Kulkarni and Padhye, 1982; Jackson et al., 1984). Because all of these have been suggested as potential sodium channel mutants, we have examined the sodium currents in neurons from each of these genotypes. Even though the known regions of sodium channel sequence similarity map to locations different from each of these mutants, analysis of their sodium currents could provide insights into additional alpha subunit genes, sirlaller subunits of the channel, or genes that are involved in the functional expression of sodium channels.

The possibility that the nap ${ }^{t s}$ mutation might affect ion channels involved in the propagation of the action potential was suggested by the observation of altered neuronal excitability in these flies. Raising the temperature resulted in an elevation in excitation threshold in both larval and adult nerve fibers in this mutant (Wu et al., 1978; Wu and Ganetzky, 1980). Effects of the napts mutation, however, are not limited to those seen at elevated temperatures. Examination of these animals at nonparalytic temperatures revealed changes in sensitivity to TTX and an increase in the refractory period of the action potential (Wu and Ganetzky, 1980). These data led to the suggestion that the $n a p^{t s}$ mutation alters the density and/or functional properties of sodium channels in axonal membranes and that the primary defect is not temperature dependent. However, they do not rule out the possibility that changes in the voltage dependence, selectivity, or gating properties of other ionic channels involved in impulse propagation could result in the observed alterations in neuronal excitability. These functional changes in channel properties could result from changes in the channel protein itself; 
Figure 1. Transient inward and sustained outward currents recorded from cultured wild-type embryonic neurons at room temperature, $24 \mathrm{hr}$ after plating. $A$, With internal potassium and external sodium as the primary charge carriers, whole-cell currents elicited by depolarizing voltage steps from -70 to $0 \mathrm{mV}$ exhibit both a transient inward component and a sustained outward component. The transient inward current is blocked by bath application of $10^{-8} \mathrm{M}$ TTX. Partial recovery of the inward sodium current 19 min after removal of the TTX is illustrated. $B$, Depolarizing voltage steps between -30 and $0 \mathrm{mV}$ from a holding potential of $-70 \mathrm{mV}$ elicit transient inward currents in the absence of outward currents when internal potassium is replaced by cesium. The sodium currents recorded under these conditions are blocked by addition of $10^{-8} \mathrm{M}$ TTX to the bathing solution.
A

Na-external; K-internal Na-external; K-internal Na-external; K-internal $\Pi \mathrm{TX}\left(10^{-8} \mathrm{M}\right)$

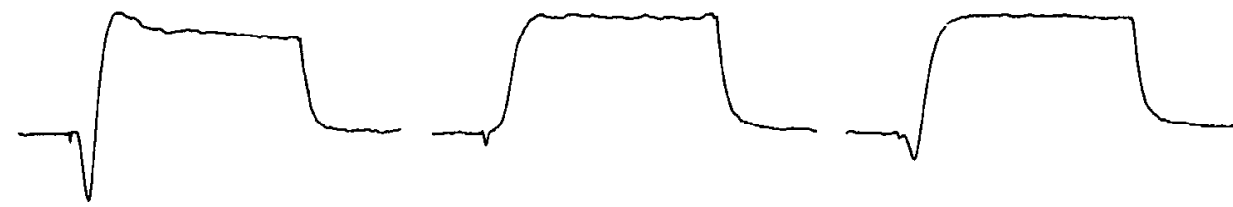

B

Na-external; Cs-internal

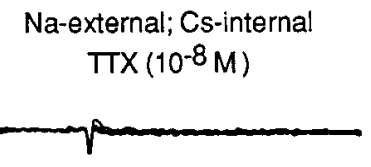

alternatively, they could reflect an alteration in the channel's environment (for example, changes in surface charge of the lipid bilayer).

Further evidence that tip- $E$ and $s e i^{t s}$ mutations, in addition to $n a p^{i s}$, specifically affect sodium channels was obtained from studies in which the binding of TTX and STX to adult CNS membrane preparations was examined. $s e i^{i s t}, t i p-E$, and $n a p^{t s}$ mutants exhibited fewer ligand biding sites compared with wild type (Kauvar, 1982; Hall et al., 1982; Jackson et al., 1984, 1985, 1986). Assuming a correlation between the number of binding sites and number of functional channels in the membrane, these findings support the contention that these mutations reduce the number of sodium channels in the membranes. seits ${ }^{\text {s. }}$ mutants did not exhibit a reduction in number of binding sites, but they did show an increase in the dissociation constant and $\mathrm{pH}$ sensitivity of binding (Jackson et al., 1984). These results suggest that one or more of these mutants could be coding for an altered gene product that affects structure of sodium channels or regulation of their number in the membrane. Though this suggestion is based on evidence obtained from larval and adult preparations, we felt that these were reasonable candidates for affecting expression of embryonic sodium currents.

In this article we characterize sodium channels in wild-type and mutant neurons by determining the current density, voltage dependence, and macroscopic kinctics of the wholc-cell sodium currents. Comparisons revealed that though the currents in wild type and mutants were similar in many respects, the average sodium current density recorded from $s e i^{i s t}$ and tip- $E$ neurons was $40-60 \%$ smaller than wild type. Thus, it appears that at least 2 of the mutants that affect neuronal excitability and toxin binding in adult and larval preparations also affect functional expression of sodium channels in embryonic neurons at room temperature.

\section{Materials and Methods}

Fly stocks. The stocks were maintained at $22^{\circ} \mathrm{C}$ on cornmeal-yeastdextrose-sucrose medium. Canton-S was used as the wild-type strain. $n a p^{t s}, s e i^{i s t}$, and $s e i^{i s 2}$ stocks were obtained from B. Ganetzky and tip-E from S. Kulkarni. Each of the stocks was tested periodically for the paralytic phenotype.

Cell culture. Cultures were prepared according to the procedures de- veloped by Seecof (1979). Midgastrula stage embryos were collected and dechorionated by immersion for $2-5 \mathrm{~min}$ in a $50 \%$ solution of bleach. Sharp micropipets were used to puncture the embryos, remove the contents, and disperse the cells onto glass coverslips. The cells were grown overnight at $22^{\circ} \mathrm{C}$ in a modified Schneiders medium (Gibco) with $20 \%$ heat-inactivated fetal calf serum (Gibco, Hazelton) and $8 \mathrm{mU} / \mathrm{ml}$ insulin (Gibco; 25-28 U/mg).

Cultures prepared from mutant embryos wcre indistinguishable from the wild-type embryo cultures. These cultures contained a number of different cell types, including myotubes and neurons (Seecof and Unanue, 1968; Seecof et al., 1971). Cultured embryonic neurons had round somata from which extended long, branching processes ending in growth cones. Cells with this morphology also stained with fluorescently conjugated antibodies to HRP, which have been demonstrated to bind to neurons in the Drosophila nervous system (Jan and Jan, 1982). Though cells distinguishable as neurons on these criteria appeared as early as 5-10 hr after culturing (Salvaterra et al., 1987), all of the recordings in this study were done $21-29 \mathrm{hr}$ after plating. Neurons chosen for physiological study generally occurred in small clumps containing $2-4$ cells.

Solutions. The composition of the solutions for experiments in which sodium and potassium currents were examined in the same cell were $\mathrm{K}$-intcrnal (in mM): $\mathrm{KF}, 70 ; \mathrm{KCl}, 70 ; \mathrm{CaCl}_{2}, 1 ; \mathrm{MgCl}_{2}, 2 ;$ EGTA, 11 ; HEPES, 10, pH 7.2; and Na-external: $\mathrm{NaCl}, 140 ; \mathrm{KCl}, 3$; TEACl, 2; $\mathrm{MgCl}_{2}, 4 ; \mathrm{CoCl}_{2}, 1 ; \mathrm{HEPES}, 5 ; \mathrm{CaCl}_{2}, 1, \mathrm{pH}$ 7.2. Sodium currents were recorded in the absence of potassium currents using solutions in which internal potassium ions are substituted by cesium ions and external potassium is omitted. Cs-internal: $\mathrm{CsCl}, 70 ; \mathrm{CsF}, 70 ; \mathrm{CaCl}_{2}, 1 ; \mathrm{MgCl}_{2}$, 2; EGTA, 11; HEPES, 10, pH 7.2, with $25 \mathrm{mM} \mathrm{NaOH.} \mathrm{Na,0K-external:}$ $\mathrm{NaCl}, 140 ; \mathrm{TEACl}, 2 ; \mathrm{MgCl}_{2}, 4 ; \mathrm{CoCl}_{2}, 1$ HEPES, 5; $\mathrm{CaCl}_{2}, 1, \mathrm{pH} 7.2$. TTX (Sigma) was added to the external solutions when indicated, at a concentration of $10^{-8} \mathrm{M}$. The osmolarity of all solutions was routinely adjusted to between 320 and $330 \mathrm{mOsm}$ with $50 \mathrm{~mm}$ sucrose.

Electrophysiology. Currents were recorded using the tight-seal wholecell recording technique (Hamill et al., 1981). Patch electrodes were coated with Sylgard and fire-polished to a resistance of 6-14 M $\Omega$ when filled with the standard solutions. Junction potentials were nulled just prior to seal formation. After establishing a high-resistance seal, the capacitance due to the pipet and membrane patch was electronically compensated to less than $0.2 \mathrm{pF}$ prior to rupture of the membrane patch. Immediately upon breaking into the cell, the whole-cell capacitance was determined by integrating the area under the capacitative transients in a current record obtained by averaging the current responses elicited by 64 depolarizing voltage steps from -70 to $-50 \mathrm{mV}$. All of the data in this study were collected from cells with whole-cell capacitances of $1-$ $5 \mathrm{pF}$. No series resistance compensation was attempted as the maximal series resistance errors from the largest sodium currents recorded were estimated to be less than $2 \mathrm{mV}$. A sodium current under good voltage control fit the following 2 criteria: (1) graded activation over a $30 \mathrm{mV}$ voltage range and (2) absence of notches in the current records. Data 
A
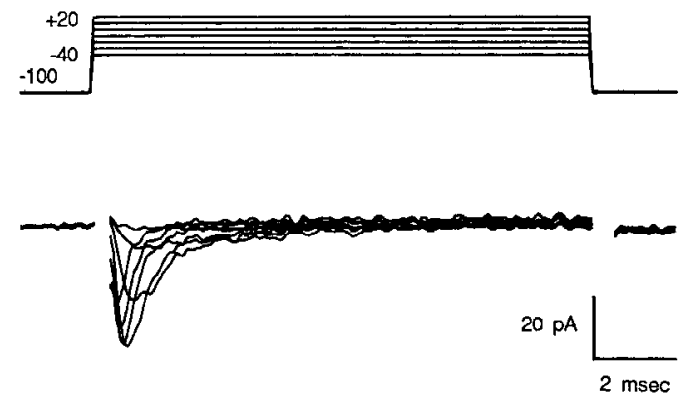

B
Command Voltage $(\mathrm{mV})$

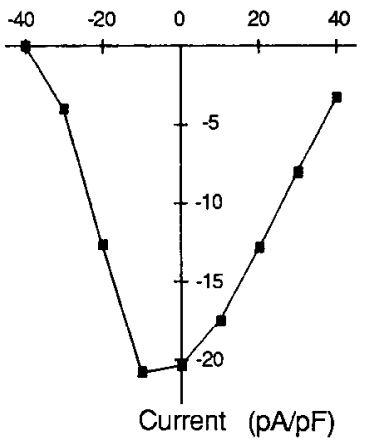

C

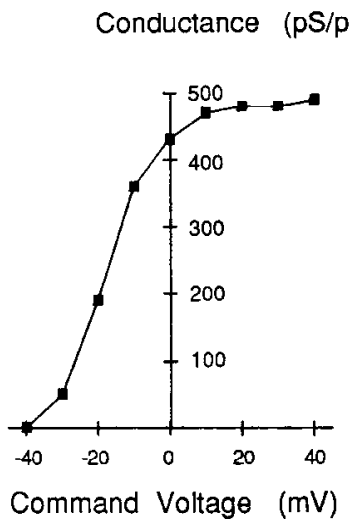

Figure 2. Sodium currents recorded from the cell body of a cultured wild-type neuron at $24 \mathrm{hr}$ and $22^{\circ} \mathrm{C}$. All of the current records in this and Figures 3 and 4 were obtained from the same cell under identical conditions. $A$, The cell was held at $-70 \mathrm{mV}$, stepped to a prepulse voltage of $-100 \mathrm{mV}$ for $500 \mathrm{msec}$, and then stepped to voltages between -40 and $+20 \mathrm{mV}$ in $10 \mathrm{mV}$ increments at $2 \mathrm{sec}$ intervals. The residual capacitative transient has been blanked at both the beginning and the end of the current trace. The traces shown were generated by averaging 4 current records at each voltage. $B$, The peak current density in $\mathrm{pA} /$ $\mathrm{pF}$ is plotted as a function of voltage. $C$, The conductance versus voltage curve for this cell was constructed using the equation of $I=g\left(E-E_{\text {rev }}\right)$ with $E_{\text {rev }}=+47 \mathrm{mV}$ (the extrapolated reversal potential from $B$ ) assuming a linear open channel $I-V$.

from cells not fulfilling both of these criteria were discarded. All currentvoltage curves and kinetic measurements were obtained from cells with an extrapolated sodium reversal potential of less than $+70 \mathrm{mV}$.

Data acquisition and analysis. Data were collected and analyzed using a List EPC-7 patch-clamp amplifier and a Digital PDP 11-73 computer. Current records were sampled at 50 or $100 \mu \mathrm{sec}$ intervals and filtered through an 8-pole Bessel filter at $2.5 \mathrm{kHz}$ unless otherwise indicated. A linearly scaled leak current, derived from the average of 64 steps between the holding potential of $-70 \mathrm{mV}$ and a test potential of $-50 \mathrm{mV}$, was subtracted from each of the current records prior to analysis. All of the experiments were performed at room temperature $\left(19-24^{\circ} \mathrm{C}\right)$. Data were collected from both wild-type and mutant flies over the same time period with identical media and recording solutions. A 2-tailed Student's $t$ test for unpaired data was used for statistical comparison of wild-type and mutant values.

\section{Results}

\section{Sodium currents in wild-type neurons}

Current separation. Voltage-clamp recordings were made from neurons between 21 and $29 \mathrm{hr}$ in culture $(24.4 \pm 0.1$, mean age \pm SEM, $n=179$ ). Both inward and outward currents were present in the majority of cells examined when internal potassium and external sodium were present as the major charge carriers. The transient inward current was reversibly blocked by $10^{-8} \mathrm{M}$ TTX, while the amplitude of the outward current
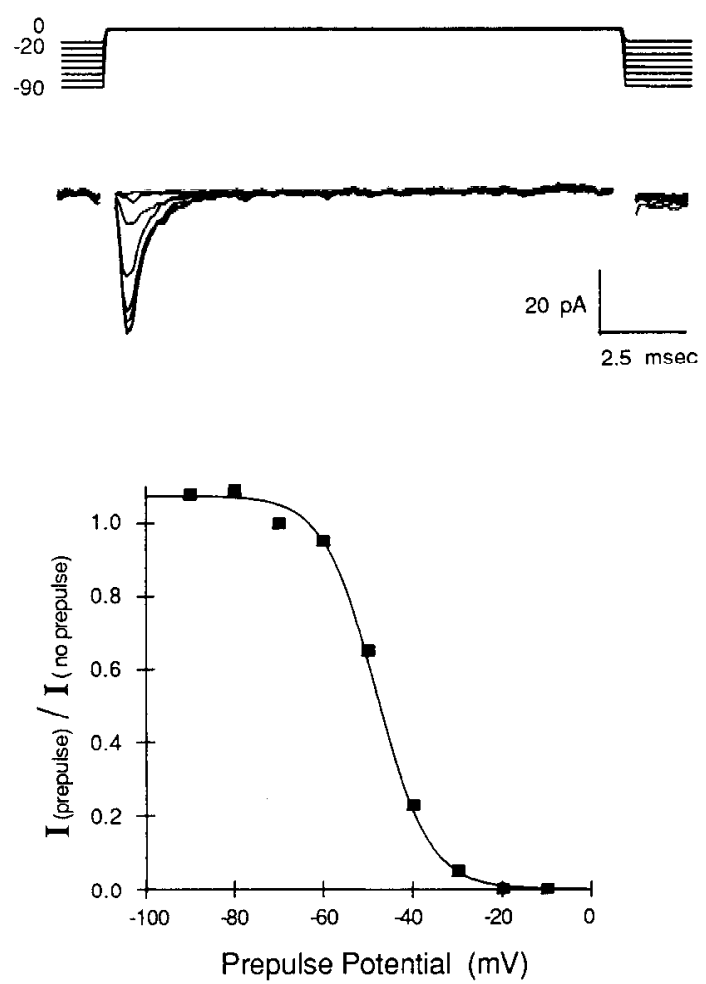

Figure 3. Steady-state inactivation of sodium currents in a wild-type neuron. $A$, Following a $500 \mathrm{msec}$ prepulse, the current amplitude in response to a test step to $0 \mathrm{mV}$ decreases as the prepulse voltage becomes more depolarized between -90 and $-20 \mathrm{mV}$. B, The peak current amplitude during the test pulse following a prepulse was normalized to the peak current amplitude elicited when the prepulse voltage was the same as the holding potential, $-70 \mathrm{mV}$. The normalized current was then plotted as a function of voltage, and the resulting data were fitted by eye with a curve generated from Equation $1 . V_{1 / 2}=-48 \mathrm{mV}$, and $z$ $=4.3$.

was not reduced by this treatment (Fig. $1 A$ ). The transient nature of the inward currents in response to maintained voltage steps, their highly voltage dependent character, and sensitivity to TTX identified these currents as voltage-dependent sodium currents. Sodium currents were recorded in isolation from potassium currents by using an internal solution containing cesium to block outward potassium currents (Fig. $1 B$ ). In these solutions, $10^{-8}$ м TTX eliminates all of the time-dependent current.

Current-voltage relation. A typical current-voltage relation $[I(V)]$ of a sodium current recorded from a wild-type neuron is illustrated in Figure 2. Sodium currents were normalized to whole-cell capacitance and expressed as current density in $\mathrm{pA}$ pF (Fig. 2B). Sodium currents in wild-type neurons were first activated around $-30 \mathrm{mV}$, peaked near $0 \mathrm{mV}$, and declined towards an extrapolated reversal potential near the calculated Nernstian equilibrium potential for sodium $(+45 \mathrm{mV})$. A conductance-voltage curve was generated assuming a linear open channel $I(V)$ and using the relation $g=I /\left(E-E_{\mathrm{rev}}\right)$, where the extrapolated reversal potential for this cell is $+47 \mathrm{mV}$ (Fig. 2C). The steep voltage dependence of the conductance at the low voltages is characteristic of sodium currents that have been cxamined in othcr organisms. The conductance begins to saturate near $-10 \mathrm{mV}$, with a maximal conductance of around $500 \mathrm{pS} / \mathrm{pF}$, reflecting the low current density seen in these neurons compared with other preparations.

Steady-state inactivation. The properties of steady-state in- 
A

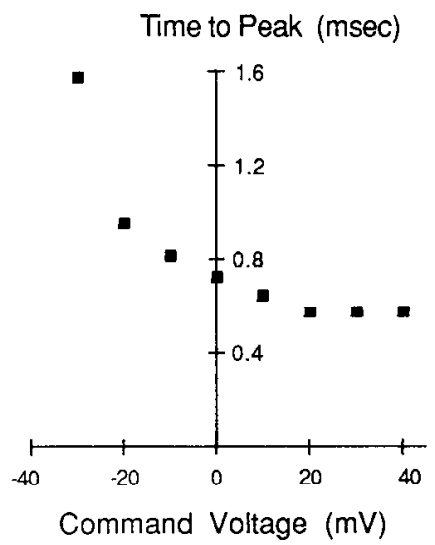

Figure 4. Time to peak current and decay time constant in a wild-type neuron. $A$, Time from the onset of the voltage step to the peak current amplitude decreases with increasing depolarization. $B$, To examine the decay of the macroscopic current, a single exponential is fitted by eye to the falling phase of the current at each voltage. $C$, Time constant of the cxponential fits (tau in msec) decreases with increasing voltage.
B

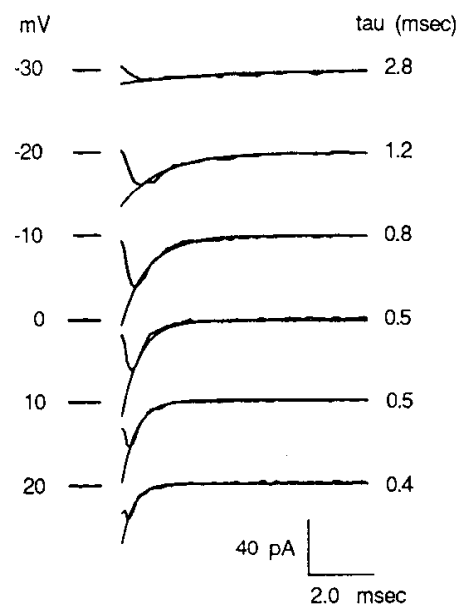

C

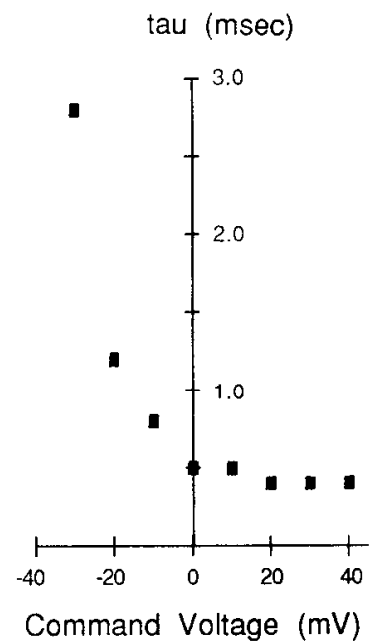

activation were examined at voltages between -90 and -10 $\mathrm{mV}$ using a $500 \mathrm{msec}$ prepulse (Fig. 3). There appears to be very little steady-state inactivation at the holding voltage of -70 $\mathrm{mV}$, while the current is almost fully inactivated by $-20 \mathrm{mV}$. A smooth curve was fitted by eye to the data using the following equation:

$$
\begin{aligned}
I / I_{0} & =\left\{1+\exp \left[\left(V-V_{\mathrm{H}_{2}}\right) / Y\right]\right\}^{-1} \\
Y & =k T / z e
\end{aligned}
$$

This equation, where $k T / e=25.4$ at roum temperature, describes the probability of a channel being available for opening (noninactivated) at the end of the $500 \mathrm{msec}$ prepulse (Hodgkin and Huxley, 1952). For this particular cell, the voltage at which half of the channels were inactivated: $\left(V_{v_{2}}\right)=-48 \mathrm{mV}$ and the equivalent gating charge: $(z)=4.3$.

Time course. The time course of the whole-cell sodium currents was characterized by the (1) time to peak, (2) decay time constant, and (3) recovery time. The time to peak current was mcasured as the delay between the onset of the voltage step and the maximal current. This value decreased with increasing voltage in a manner typical of voltage-gated sodium currents (Fig. $4 A$ ). Though series resistance did not present significant errors in determining the true command potential, it is possible that it did affect the time course of the currents. The settling time of the clamp with a $50 \mathrm{G} \Omega$ head-stage resistor and a $2.5 \mathrm{pF}$ cell was typically $0.5-1 \mathrm{msec}$ in response to a $20 \mathrm{mV}$ depolarizing step from the holding potential. This could distort the time course of the currents between 0 and $1 \mathrm{msec}$, and thus the values determined for time to peak current at the higher voltages may be subject to some uncertainty. However, the error should be similar in all of the genotypes tested as the mean cell capacitance did not vary between mutants.

The falling phase of the inward current can be well fitted by a single exponential (Fig. 4B). The time constants of these exponential fits were used to characterize the voltage dependence of the macroscopic current decay. The rate of decay increased with increasing depolarizations between -20 and $+40 \mathrm{mV}$ (Fig. $4 C)$.

Recovery from inactivation was examined using a 2 pulse protocol in which a conditioning voltage step from -70 to 0 $\mathrm{mV}$ was followed by a test pulse of identical amplitude but varying latency. As can be seen in Figure $5 A$, only a small fraction of the total number of channels is available for activation in response to the second voltage step after short interpulse intervals $(<5 \mathrm{msec})$. Increasing interpulse intervals resulted in successively larger currents elicited during the test (second) step to $0 \mathrm{mV}$ (Fig. 5). The normalized current (current amplitude in response to test pulse divided by the current amplitude elicited by the conditioning pulse) elicited by the second step was examined over a range of interpulse intervals between 

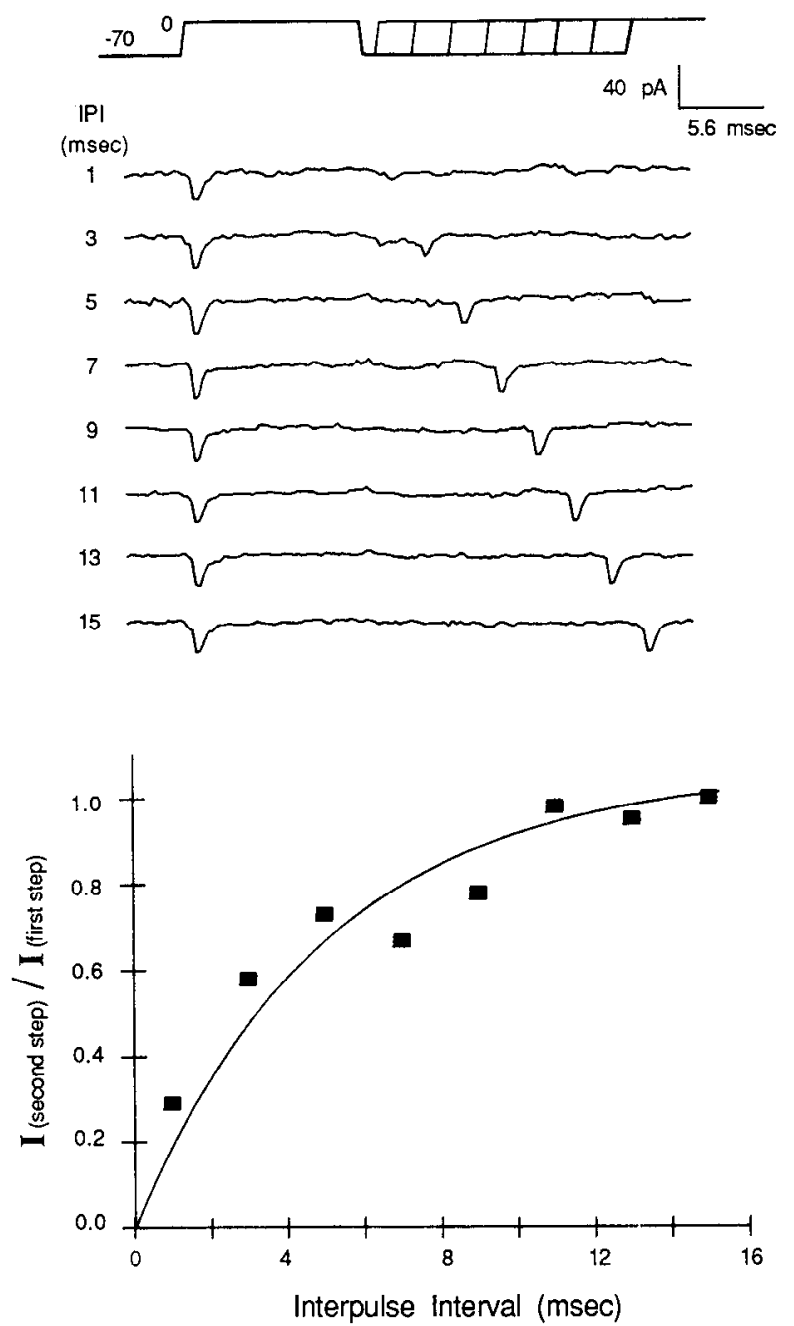

Figure 5. Recovery from inactivation. $A$, Recovery from inactivation was examined using the 2 pulse protocol illustrated with a test voltage of $0 \mathrm{mV}$ and a recovery voltage of $-70 \mathrm{mV}$. The current records were sampled at $400 \mu \mathrm{sec}$ intervals. The current amplitude elicited by the test step increases with increasing interpulse intervals (IPI) between 1 and 15 msec. $B$, Current amplitude elicited by the second test step was normalized to that from the first step in each trial and plotted as a function of interpulse interval. A single exponential with a time constant of $4.6 \mathrm{msec}$ was fitted by eye to the data.

1 and $20 \mathrm{msec}$. The time course of this recovery process was described by a single exponential (time constant $=4.6 \mathrm{msec}$ ) fitted by eye to the data points (Fig. 5).

\section{Sodium currents in mutant neurons}

Current separation. Both inward and outward currents could be clicitcd in neurons from all of the genotypes examined approximately $24 \mathrm{hr}$ after plating, in the presence of internal potassium and external sodium (Fig. 6). The outward current was blocked when cesium replaced internal potassium as illustrated in records obtained from a napts mutant in Figure 6C. As demonstrated for wild type, transient inward currents in the mutant genotypes were blocked by $10^{-8} \mathrm{M}$ TTX, while the outward currents were not affected (Fig. 6, $A, B$ ). The outward currents in these cells were similar to those seen in wild-type neurons.

Isolated sodium currents from all 4 of the mutant genotypes examined and from a wild-type neuron are shown in Figure 7. There was some variability in both the steady-state and kinetic

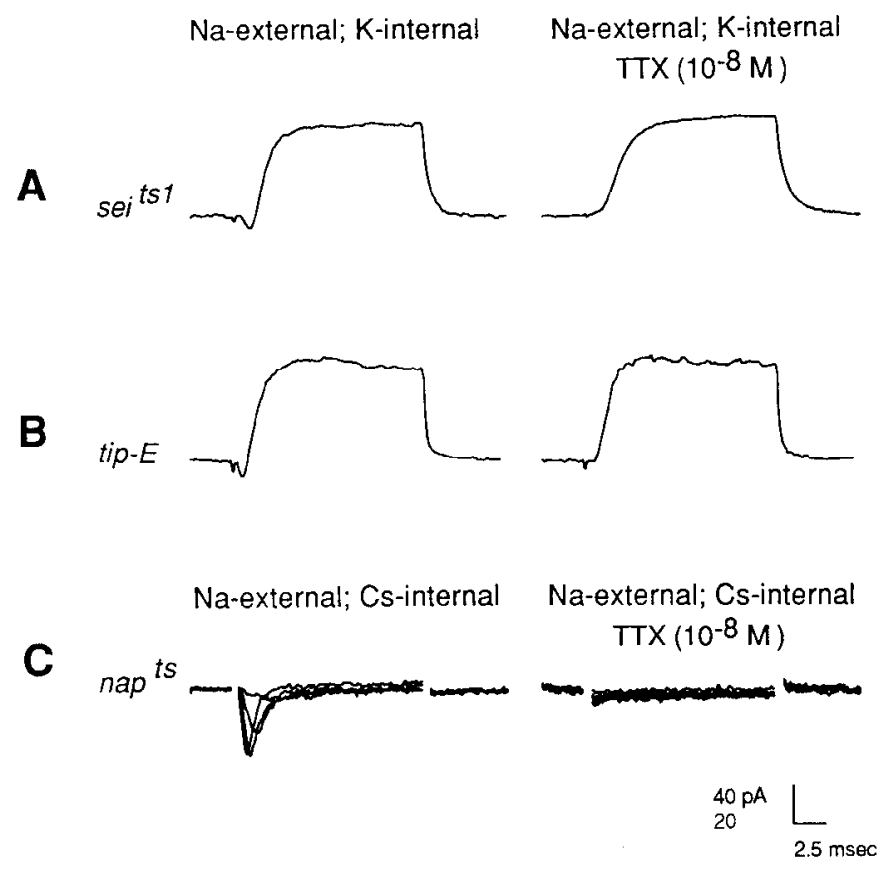

Figure 6. Inward and outward currents arc present in neurons from all of the temperature-sensitive paralytic genotypes when examined at approximately $24 \mathrm{hr}$ and $22^{\circ} \mathrm{C}$. Similar to the wild-type cells, the inward currents are blocked by $10^{-8} \mathrm{M}$ TTX, and the outward currents are eliminated when cesium replaces potassium in the internal medium. $A$, Averaged current responses from 8 voltage steps from -70 to $0 \mathrm{mV}$ in the presence and absence of $10^{-8} \mathrm{M}$ TTX in a seits neuron. $B$, Single current traces in response to voltage steps from -70 to $0 \mathrm{mV}$ in a neuron from a tip-E embryo. $C$. Transient inward currents elicited by a series of voltage steps between -30 and $0 \mathrm{mV}$ when the outward currents were blocked by internal cesium in a nap ${ }^{s}$ neuron.

parameters of the sodium currents from every genotype, including wild type. Therefore, examination of possible differences between genotypes necessitated comparisons of averaged curves describing the voltage dependence and time course of sodium currents obtained from a number of neurons from each of the different genotypes. As illustrated in Figure 8, the average current-voltage relation for the population of wild-type neurons was very similar to that seen in individual wild-type neurons (Fig. 2). To assess whether any of the mutants exhibited changes in the current density or voltage dependence of the sodium currents the averaged $I(V)$ curves generated for each of the genotypes were compared to the wild-type values.

Current-voltage relations in mutants: napts. Comparison of the mean $I(V)$ curve generated in cells of the $n a p^{t s}$ genotype reveals a curve that is very similar in both amplitude and shape to that from wild-type cells (Fig. $8 \mathrm{~A}$ ), indicating that the current density and voltage dependence of the sodium currents are not significantly altered by the napis mutation.

$s e i^{i s t}$ and $s e i^{i s 2}$. We also examined the current-voltage relation of sodium currents in neurons of flies with mutations at the sei locus. In $s e t^{t s}$ neurons the current density was $40-60 \%$ smaller than wild type at each voltage between -30 and $+40 \mathrm{mV}$ (Fig. $8 B$ ). It is unlikely that this low current density is a consequence of a change in the voltage dependence of the current given the similarity in the shape of the mutant and wild-type curves. A smaller number of channels and/or single-channel conductance could contribute to the low current density. Another allele of the sei locus, seits ${ }^{i 2}$, exhibited no significant change in either the current density or voltage-dependence (Fig. $8 B$ ). 

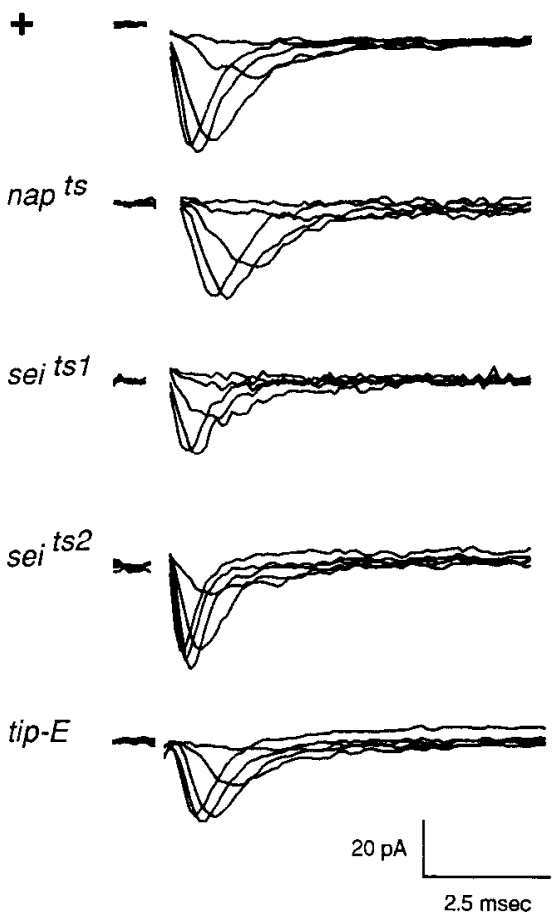

Figure 7. Sodium currents can be recorded in all of the genotypes examined. Shown are examples of sodium currents from individual cells from each of the genotypes in response to depolarizing voltage steps between -30 and $+10 \mathrm{mV}$ in $10 \mathrm{mV}$ increments from a holding voltage of $-70 \mathrm{mV}$. The residual capacitative transient has been blanked at the beginning of each trace.

tip- $E$. The sodium current density at voltages between -10 and $+40 \mathrm{mV}$ in tip- $E$ neurons was $40-60 \%$ smaller than wild type (Fig. $8 \mathrm{C}$ ). As discussed for $s e i^{i s l}$, it is possible that changes in the number of channels or the single-channel conductance could be responsible for the lower whole-cell sodium current density. However, it was also noted in tip- $E$ cells that at the lower voltages ( -30 and $-20 \mathrm{mV}$ ) the current density is similar to wild type. This leaves open the possibility that a slight alteration in the voltage dependence of the sodium current in tip- $E$ neurons may contribute to, or occur in addition to, the reduction in sodium current density.

Steady-state inactivation. The possibility that some of the mutations might result in changes in the inactivation properties of the sodium channels was investigated initially by examining the properties of steady-state inactivation in each of the genotypes. The mean values of the normalized currents at each prepulse potential were plotted as a function of prepulse voltage for 7 or more cells from each geneotype (Fig. 9A). As can be seen, values for all of the genotypes were similar over the entire voltage range. Fits of equation 1 (by eye) to the data had a $V_{1 / 2}$ $=-40.3 \mathrm{mV}$ and an equivalent gating charge $z=4.3$. Similar fits were made to the individual cells in each genotype, and a comparison of the mean values for determinations of both $V_{1 / 2}$ and $z$ is shown in Table 1 .

Time course of sodium currents. Mutations affccting the gating properties of the sodium channels might be expected to alter the time course of the macroscopic current. The mean values for time to peak current were examined at voltages between -30 and $+40 \mathrm{mV}$ for each of the genotypes examined (Fig. 9B). No significant differences were found when the data from $s e i^{i s l}$, $s e i^{i s 2}$, and $n a p^{t s}$ were compared with wild type. At the higher voltages, between 0 and $+40 \mathrm{mV}$, the values for tip- $E$ currents appear to be somewhat slower $(\sim 300 \mu \mathrm{sec})$ than the wild type $(p \leq 0.05)$. Time constants of exponential fits to the decay of the sodium current were similar in all of the mutants examined (Fig. 9C). The time course of recovery from inactivation at -70 $\mathrm{mV}$ as investigated with the 2 pulse protocol described previously was also similar in the 4 mutants examined (Fig. 9D). Based on these analyses there are no significant differences in the macroscopic kinetics of $s e t^{i s}$ or nap ${ }^{i s}$ mutants compared with wild type. Variability of the values might preclude accurate identification of subtle kinetic changes in these genotypes. The only change in kinetics was observed in tip- $E$, which exhibited
A

Command Voltage $(\mathrm{mV})$

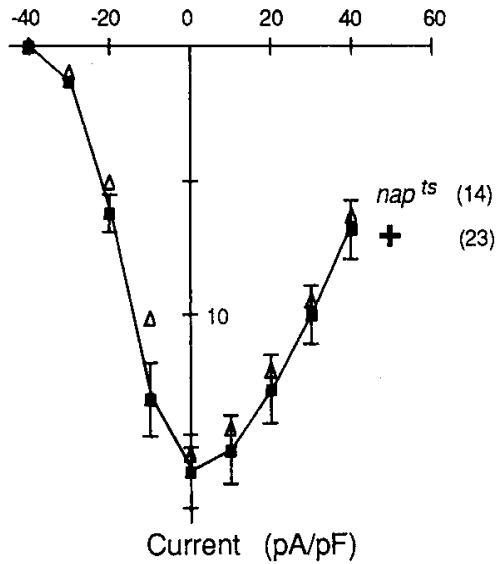

B Command Voltage $(\mathrm{mV})$

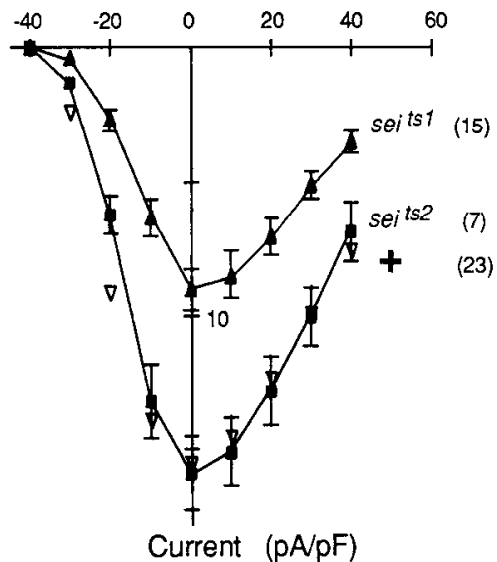

C Command Voltage $(\mathrm{mV})$

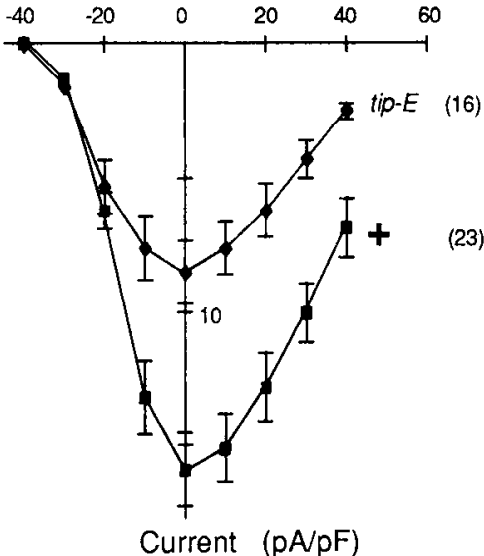

Figure 8. Comparison of the mean current density versus voltage curve compiled from the populations of wild-type and 4 mutant genotypes. Each point represents the mean current density value at that voltage for the indicated number of cells from each of the genotypes. $A$. Data for nap ${ }^{t s}$ $(\triangle)$ are very similar to the curve described by the values from wild-type $(\square)$ cells. The bars indicate the (SEM) for the wild-type values. $B$, Comparison of $\operatorname{set}^{i s t}(\mathrm{~A})$ data points reveals a $40-60 \%$ decrease in current density at each of the voltages examined. The values between -30 and $+40 \mathrm{mV}$ were found to be significantly different at the $p=0.05$ level (Student $t$ test, 2-tailed, unpaired). In contrast, there is no significant difference in the $I-V$ curves generated for seisz $(\nabla)$ neurons. $C$, A decrease in the current density is seen in tip- $E(\$)$ neurons when values between -10 and +40 are compared with wild type ( $\square$. These values were different at the $p=0.05$ level (Student $t$ test, 2-tailed, unpaired). 
A

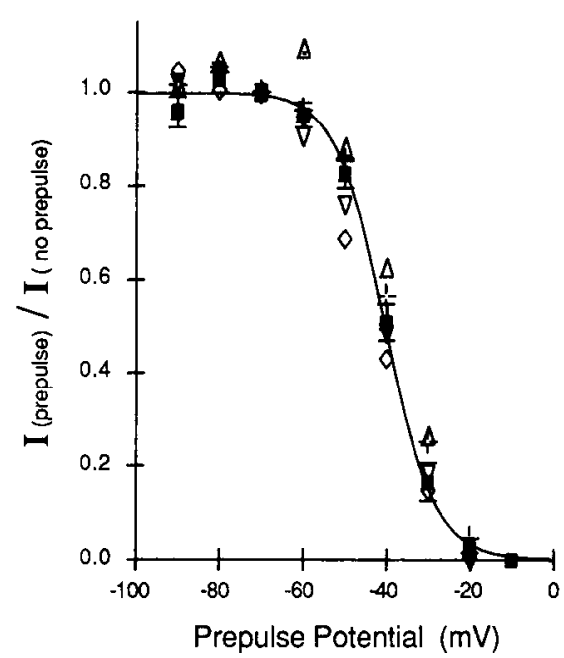

C

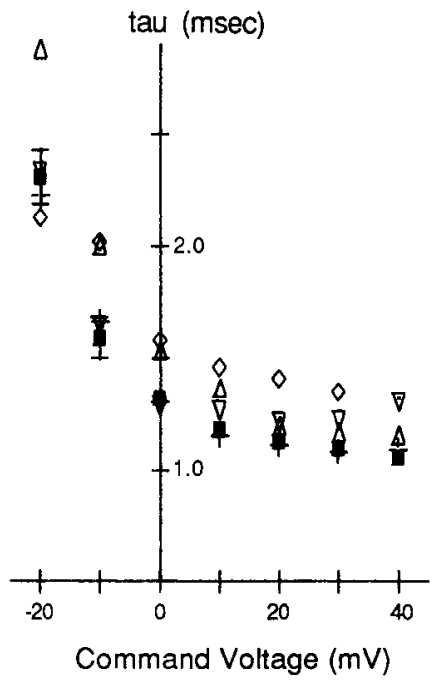

B

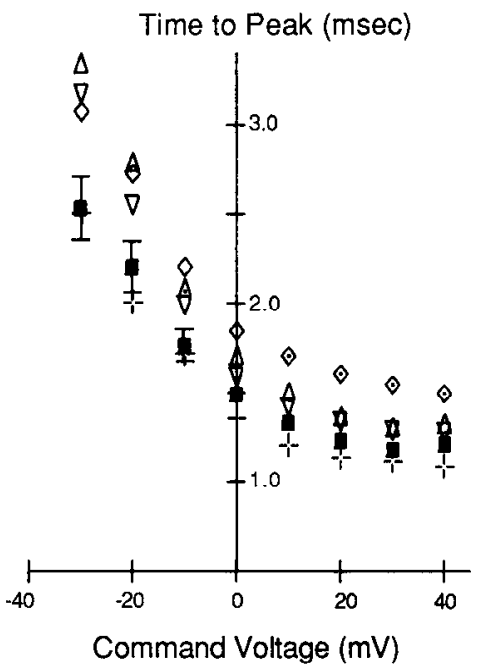

D

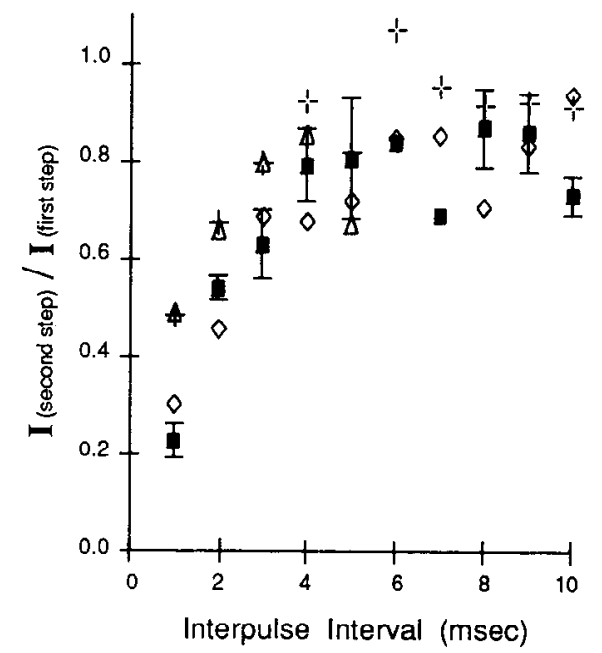

Figure 9. Comparison of steady-state inactivation and macroscopic kinetic properties of mutants with wild type. Each symbol indicates the mean value for 7 or more cells in each of the populations examined with the exception of $D$, where values from 3 cells were used to determine the mean. Symbols: wild type, $\square$; nap $^{t s}$, +; seits ${ }^{i s l} \Delta ; \operatorname{sel}^{i s 2}, \nabla$; and tip- $E, \diamond$. The wild-type values are accompanied by bars indicating the SEMs. $A$, Steady-state inactivation is similar in all of the genotypes examined. A curve generated from Equation 1 was fitted by eye to the data with a $V_{1 / 2}=-40.3$ and $z=4.3 . B$, Mean values for time to peak current in the 4 mutant genotypes are compared with the wildtype values. Symbols with dots in the middle indicate mean values that are significantly different from wild type $(p=0.05)$. These include values for tip- $E$ at +10 to $+40 \mathrm{mV}$ and seitst at 20 and $-10 \mathrm{mV}$. C, Time constants of exponential fits to the decay phase of the currents in mutant and wild-type cells are compared between -20 and $+40 \mathrm{mV}$. The only point that is significantly different than wild type is seitsz at $+40 \mathrm{mV}$. $D$, Time course of recovery from inactivation appears similar in all of the genotypes examined. a slightly slower time to peak current than wild type at the higher voltages.

Examination of sampling bias. Based on these data it is clear that mutations in both the tip-E and seizure loci can reduce the sodium current density in the cultured embryonic neurons when they are examined approximately $24 \mathrm{hr}$ after plating. Though there are no reports of alterations in life cycle duration in any of the mutants examined, it was important to consider the possibility that a delay in embryonic development of either tip- $E$ or $s e i^{i s t}$ could contribute to the reduced amplitude of sodium currents observed in these cultures. Therefore, time-lapse video recordings of single embryos were used to determine the timing of pole cell formation, posterior midgut invagination, first body movements, and hatching. Both seitsl and tip-E embryos showed a normal time course of development compared with wild-type embryos. The morphology of cells in mutant cultures also appears similar to wild type at $20-30 \mathrm{hr}$ of development. In addition, as no significant decrease in the outward current density at $0 \mathrm{mV}$ from a prepulse potential of $-70 \mathrm{mV}$ was observed in $\operatorname{sei}^{i s t}(n=3)$ or tip-E $(n=8)$ neurons compared with wild type,

Table 1. Voltage dependence of resting inactivation for wild-type and mutant genotypes

\begin{tabular}{lccccc} 
& + & nap & $s e i^{i s}$ & sei $^{i s 2}$ & tip-E \\
\hline$V_{1 / 2}(\mathrm{mV})$ & $-40 \pm 1$ & $-40 \pm 2$ & $-38 \pm 2$ & $-43 \pm 3$ & $-45 \pm 4$ \\
Charge & $4.2 \pm 0.2$ & $4.3 \pm 0.2$ & $4.4 \pm 0.3$ & $3.8 \pm 0.3$ & $3.7 \pm 0.2$ \\
$n$ & 15 & 11 & 7 & 7 & 7
\end{tabular}

Inactivation curves from individual cells were fitted with equation 1 . This table presents the mean values for $V_{1 / 2}$ and $z$ made in each of the 5 genotypes examined. 
A

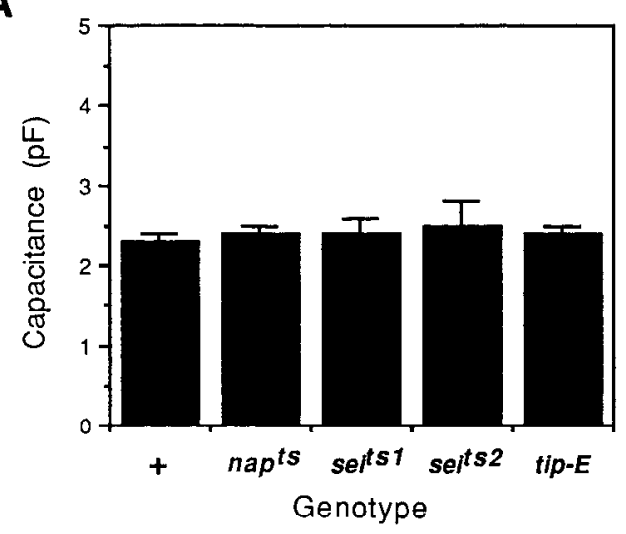

B

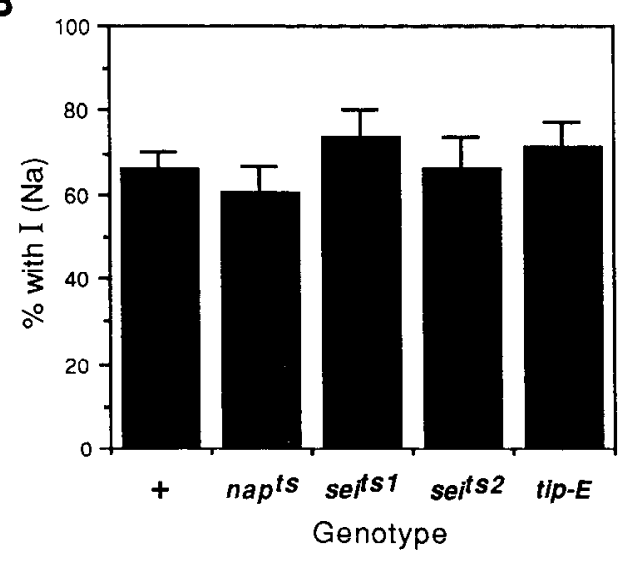

C

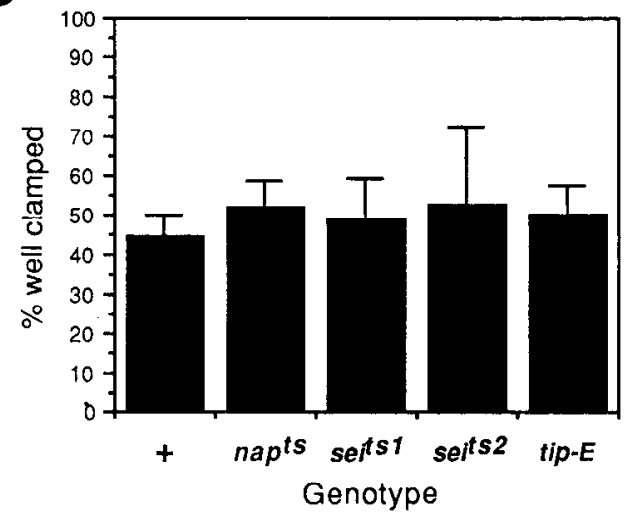

Figure 10. Three parameters defining the population of cells in cultures prepared from the different genotypes. $A$, Average whole-cell capacitance (in $\mathrm{pF}$ ) was determined in each of the genotypes from the total population of cells examined. Bars in all cases indicate SEMs. $B$, Mean percentage of cells expressing sodium currents calculated from each plating for all 5 genotypes. $C$, Of the cells expressing sodium currents the percentage from which well-clamped sodium currents were obtained; again, this was determined on a per plating basis.

it appears that at least the potassium currents develop along a normal time course. However, this does not rule out the possibility that these mutations specifically delay the development of neuronal sodium currents.

The heterogeneity of the cultures in terms of cell type also leaves open the possibility that different populations of neurons were sampled for voltage-clamp experiments in the different genotypes. This may contribute to the apparently low mean current density observed in $t i p-E$ and $s e i^{i s t}$ neurons. Therefore, to more carefully define the neuronal population sampled, we compared (1) whole-cell capacitance, (2) the percentage of cells examined that expressed sodium currents, and (3) the percentage of cells generating a sodium current that could be adequately voltage-clamped in each of the genotypes examined with wild-type values. From a total of 179 wild-type neurons examined in 35 separate platings, the mean cell capacitancc was $2.3 \pm 0.1 \mathrm{pF}$ (mean $\pm \mathrm{SEM}$ ). The percentage of cells with sodium currents in any given plating was $66 \pm 4 \%$ (mean \pm SEM). Of the cells with sodium current, only $42 \%$ were considered to be under good voltage control. No significant difference in these 3 measures was apparent in any of the genotypes (nap ${ }^{2 s}$, $s e i^{i s t}, s e i^{t s z}$, and $t i p-E$ ) (Fig. 10).

\section{Discussion}

The results presented here demonstrate that cultured embryonic neurons are a suitable preparation for a combined biophysical, genetic, and molecular study of sodium channels in Drosophila. The properties of the sodium currents in cultured wild-type neurons can be examined under voltage-clamp conditions and are very similar to those that have been reported in a variety of other vertebrate and invertcbratc preparations, although the current density is low compared with many excitable tissues (see Hille, 1984). In addition, changes in neuronal sodium current expression have been observed at room temperature in cultures prepared from mutant genotypes.

The cells chosen for physiological study were a subset of the total neuronal population arising from the plating of dissociated, gastrulating embryos. These neurons had round cell bodies, generally occurred in clusters of 2-4 cells, and extended long, branching processes of varying length. Cells with wellclamped sodium currents were not morphologically distinguishable from those without any sodium currents or those that were poorly clamped. As we quantitatively examined only sodium currents under good voltage control, it seems likely that the majority of the sodium channels characterized in this study are located in or near the soma. Outward currents, which could be blocked by internal cesium, were also present in a majority of these neurons.

In order to assess directly the effect of putative sodium channel mutants ( nap $^{t s}$, seitsl ${ }^{i s} e^{i s 2}$, and tip-E) on neuronal sodium channel expression, we compared the properties of sodium currents recorded from mutant and wild-type neurons. From these studies we have identified 2 genes, seits and tip- $E$, in which mutations can alter the normal expression of sodium currents in embryonic Drosophila neurons. In contrast, the nap ts mutation does not appear to alter the expression of sodium currents in these cells. Outward currents, whole-cell capacitance, percentage of cells expressing sodium currents, and percentage of the cells that could be well clamped were similar in all of the 4 mutant genotypes compared with wild type.

Our results confirm the previous conclusion that mutations at both the sei and tip- $E$ loci affect voltage-dependent sodium channels and extend the known effects of these mutations to the embryonic stage. In addition, they document that, although the behavioral phenotype is a temperature-sensitive paralysis, alterations in sodium channel expression occur at room temperature. Given that wild-type flies also paralyze at elevated tem- 
peratures $\left(40^{\circ} \mathrm{C}\right)$, though with a slower time course than the mutants (Jackson et al., 1985), it is possible that a simple reduction in the number of sodium channels in these mutants results in an increased sensitivity to temperature-induced blockade of the action potential. Examination of both action potentials and sodium currents in neurons at high temperatures may reveal additional differences between mutant and wild-type sodium currents and the mechanism of spike failure. These studies are difficult due to the small amplitude and fast kinetics of sodium currents even at room temperature. However, the alterations in sodium current density that we have documented at $19-24^{\circ} \mathrm{C}$ in 2 of the mutant genotypes substantiate the usefulness of voltage-clamp experiments on embryonic neurons at room temperature for the study of genes important in the expression of sodium currents.

In light of our results on the sei and tip- $E$ loci, the lack of effects of the napts mutation on sodium channel expression is somewhat surprising. The napts behavioral phenotype is very similar to that of se $i$ and $t i p-E$, and alterations in physiological properties and toxin binding have been reported that are consistent with this mutation also affecting sodium channel expression. Among the possibilities for this seeming discrepancy are (1) nap may affect sodium channels only at temperatures that are behaviorally restrictive (this is unlikely because of the welldocumented phenotypes in nap flies at room temperature), (2) it is possible that nap affects a class of sodium channels that are not expressed in embryos, or only exerts its effects at later stages, (3) nap may affect sodium channels that are present in axons but not in cell bodies, and (4) nap may affect excitability by some mechanism other than through sodium channels (although this would be inconsistent with the toxin binding results). It is clear that these results, taken with the finding of multiple genes in the Drosophila genome with homology to the vertebrate sodium channel (see below), indicate that developmental and tissue-specific regulation of sodium channels in flies may be complex.

\section{seits $^{\text {ts }}$ and tip-E mutants}

The sodium current density in both $s e i^{t s l}$ and tip- $E$ was $40-60 \%$ smaller than wild type when examined at ambient temperature. The other steady-state and kinetic properties of the sodium currents in these cells appeared similar to wild-type values. The $40-60 \%$ reduction in current density that we report is consistent with the results from binding studies, which documented a decrease of similar magnitude $(5-37 \%$ and $30-50 \%)$ in the number of STX binding sites in adult head membrane preparations for $s e i^{i s t}$ and $t i p-E$, respectively (Jackson et al., 1985, 1986). The small number of binding sites in conjunction with the present results indicating a low sodium current density in tip-E and $s e l^{i s t}$ support the hypothesis that these mutations result in a reduction in the number of functional sodium channels in the membrane. The lack of a significant reduction in the peak current density at both -30 and $-20 \mathrm{mV}$ and the slight difference in the time to peak current in tip-E are also consistent with the possibility that a small change in the voltage-dependent gating might contribute to the low current density observed in these mutants. However, these data do not rule out the possibility that there is a change in single-channel conductance or a slow inactivation of channels contributing to the low current density.

Could delays in the development of $t i p-E$ and $s e i^{t s i}$ embryos account for the small current density observed in neurons when examined $24 \mathrm{hr}$ after plating? The cultures of mutant and wildtype embryos were indistinguishable at $24 \mathrm{hr}$, having differentiated both neurons and myotubes of similar morphologies. We also examined the timing of the organismal development in these genotypes from fertilization to hatching and did not observe any difference compared with wild-type development. We did not address the question of whether the mutant neurons in culture exhibit changes in sodium current densities along the same time course. However, we did not find significant differences in potassium current density when examined at the same time that reductions in the sodium current are apparent.

\section{$\operatorname{sei}^{i s^{2}}$ mutant}

Sodium current density was unaffected by the $s e i^{i s 2}$ mutation. These results are consistent with previous studies, which reported that the number of STX binding sites in $s e i^{i s 2}$ CNS membranes was similar to wild type. In these same binding studies changes in the dissociation constant and pH sensitivity of STX binding were also noted, leading to the suggestion that this mutation caused a structural alteration in the sodium channels (Jackson et al., 1984). Such an alteration in an ion channel protein may cause changes in the voltage dependence or kinetics of the sodium currents. However, we did not observe any evidence of alterations in these properties of the $\operatorname{sei}^{i s 2}$ sodium currents. The lack of a correlation between alterations in the binding affinity of toxins which specifically interact with sodium channels and changes in the functional properties of sodium channels has precedence. A clone of variant neuroblastoma cells resistant to the cytotoxic effect of scorpion toxin and exhibiting low affinity for the toxin, express sodium channels with normal functional properties as assessed in whole-cell voltage-clamp experiments (Gonoi et al., 1984). In light of the changes in the $K_{D}$ of STX binding in membrane extracts from $\operatorname{set}^{i s 2}$, it would be interesting to examine the I'IX sensitivity of sodium currents in cultured $s e i^{i s 2}$ neurons. Unfortunately, because of the slow washout of TTX compared with the life of the cell in whole-cell clamp, this type of experiment is difficult. In addition, it is unlikely that we could resolve a 2 -fold change in sensitivity.

Recent electrophysiological studies on adult $s e i^{t s l}$ and $s e i^{i s 2}$ mutants have reported that a shift in temperature from 22 to $45^{\circ} \mathrm{C}$ induced spontaneous activity in the giant fiber system (T. Elkins and B. Ganetzky, personal communication; Kasbekar et al., 1987). It does not seem likely that a simple reduction in sodium current density in the adult axons, such as reported here for $s e i^{i s t}$ neurons (no difference noted for $s e i^{i s 2}$ ) could account for this phenomenon, although a preferential effect on inhibitory neurons could lead to spontaneous activity. These findings again raise the possibility that separate populations of cells may be differentially regulated (embryonic vs. adult channels; axonal vs. somal channels). Examination of sodium currents in sei mutants at elevated temperatures may reveal additional changes that could contribute to the increase in spontaneous activity.

The differences in the effects on sodium current density of the 2 sei alleles is not unexpected given the fact that the gene dose dependence of paralysis, the general behavior of the mutants, and their toxin binding also differ (Jackson et al., 1984, 1985; Kasbekar et al., 1987). These differential effects illustrate the value of studying a number of alleles of any particular locus. Analysis of single sodium channels will be critical in determining the mechanisms by which sei ${ }^{i s t}$ and $t i p-E$ mutations result in a reduction in sodium current density in the cultured neurons. 


\section{nap ${ }^{\text {ss }}$ mutant}

Sodium currents in embryonic neurons cultured from nap ${ }^{t s}$ mutants are indistinguishable from wild type in density, voltage dependence, and kinetics of the macroscopic current. This demonstrates that nap $p^{t s}$ mutants have at least one gene coding for functional sodium channels that is regulated and expressed in a manner similar to wild type. The results from the present study are not the first to suggest that the properties of the sodium currents in some nap ${ }^{t s}$ cells are similar to wild type. Two recent physiological studies have revealed that conduction, in at least some of the axons in the cervical giant fiber pathway of adult nap ${ }^{\text {ss }}$ flies, is not blocked even at temperatures that induce paralysis (Nelson and Baird, 1985). This conduction is TTX sensitive, suggesting that these flies have some functional sodium channels that are not blocked at elevated temperatures(T. Elkins and B. Ganetzky, personal communication). This raises the possibility that the nap ${ }^{2 s}$ mutation affects expression of sodium currents differently in different populations of cells. All of the prior studies examining action potential propagation (Wu et al., 1978; Wu and Ganetzky, 1980) and toxin binding (Hall et al., 1982; Jackson et al., 1984), which suggested that naps affects sodium channels, were performed in larval and adult preparations as opposed to the present study, which focused exclusively on sodium currents in embryonic neurons. The nap ${ }^{s s}$ mutation could affect genes that are important for development of postembryonic sodium currents but that are unnecessary for embryonic sodium current expression. Additionally, the sodium channels that have been examined in the present study are most likely present in or near the soma of the embryonic neurons, whereas previous physiological studies examined sodium currents involved in axonal conduction. If there are sodium channel subtypes specifically located in the axonal membrane, they may be regulated differently than those in the cell body. The existence of multiple sodium channel genes in rat brain (Noda et al., 1986) and differential expression of functionally distinct classes of sodium channels in developing rat muscle (Weiss and Horn, 1986) support the possibility that expression of sodium currents can be complex.

\section{Sodium channel genes in Drosophila}

Our results do not indicate whether the deficits in sodium channels density in the sei and $t i p-E$ mutants are due to structural or regulatory mutations. Changes at the level of transcription, translation, or postranslational modifications of molecules important for sodium current expression are all candidates for the site of action of these mutations. Analysis of single sodium channels will be critical in determining the mechanisms by which these mutations result in a reduction in sodium current density in the cultured neurons. They will not help, however, in distinguishing between structural and regulatory mutations, as a change in single-channel conductance or in the number of channels per cell is consistent with either mechanism. A combination of further electrophysiological and molecular studies will be needed to determine the properties of each gene involved in the function of fly sodium channels.

Recent progress has been made in the cloning of genes that have high sequence similarity to the vertebrate sodium channel alpha subunit (Salkoff et al., 1987; K. Loughney and B. Ganetzky, personal communication). Neither of the 2 reported sequences maps to the chromosomal locations of the mutants examined in this study. There is evidence, however, that both of the genes with sequence similarity to the alpha subunit are expressed in embryos. Northern blot analysis has shown that mRNA homologous to the putative alpha subunit gene locatcd in chromosome region 60D-E is present in embryos (Salkoff et al., 1987), but voltage-clamp experiments on cultured embryonic neurons deficient for this region do not abolish sodium channel expression (S. Germeraad, D. O'Dowd, and R. Aldrich, unpublished observations). In contrast, some mutant alleles of the para $^{t s}$ locus, which has recently been shown to have high sequence similarity to the vertebrate sodium channel alpha subunit (K. Loughney and B. Ganetzky, personal communication), drastically reduce the expression of sodium currents in embryonic neurons (O'Dowd et al., 1987). The fact that these para alleles can completely eliminate sodium currents in a large fraction of the cells raises the possibility that it may be the primary gene coding for sodium channels in these cell bodies under our experimental conditions. Further work on both of these loci at a number of different developmental stages will be necessary for the understanding of the function and regulation of Drosophila alpha subunit genes. The specific role of beta subunits in sodium channel function is unclear in vertebrates (see Agnew, 1984; Catterall, 1986; Krafte et al., 1987; Stuhmer et al., 1987) and has not been addressed with regards to the fly sodium channels. Sei and tip-E could conceivably code for these smaller subunits or could be involved in the assembly or regulation of channels that are coded for by other distinct loci.

\section{References}

Agnew, W. S. (1984) Voltage-regulated sodium channel molecules. Annu. Rev. Physiol. 46: 517-530.

Byerly, L. (1985) Voltage-dependent currents in embryonic cultures of Drosophila neurons. Soc. Neurosci. Abstr. 11: 149.

Catterall, W. A. (1986) Molecular properties of voltage-sensitive sodium channels. Annu. Rev. Biochem. 55: 953-985.

Ganetzky, B., and C.-F. Wu (1986) Neurogenetics of membrane excitability in Drosophila. Annu. Rev. Genet. 20:13-44.

Gonoi, T., B. Hille, and W. A. Catterall (1984) Voltage clamp analysis of sodium channels in normal and scorpion toxin-resistant neuroblastoma cells. J. Neurosci. 4: 2836-2842.

Hall, J. C. (1982) Genetics of the nervous system in Drosophila. Q. Rev. Biophys. 15: 223-479.

Hall, L. M., S. D. Wilson, J. Gitschier, N. Martinez, and G. R. Strichartz (1982) Identification of a Drosophila melanogaster mutant that affects the saxitoxin receptor of the voltage-sensitive sodium channel. CIBA Found. Symp. 8: 207-220.

Hamill, O. P., A. Marty, E. Neher, B. Sakmann, and F. Sigworth (1981) Improved patch-clamp techniques for high resolution current recording from cells and cell free membrane patches. Pfluegers Arch. 391 . 85-100.

Hillc, B. (1984) Ionic Channels of Excitable Membranes, pp. 205225, Sinauer, Sunderland, MA.

Hodgkin, A. L., and A. F. Huxley (1952) The dual effect of membrane potential on sodium conductance in the giant axon of Loligo. J. Physiol. (Lond.) 116: 497-506.

Jackson, F. R., S. D. Wilson, G. R. Strichartz, and L. M. Hall (1984) Two types of mutants affecting voltage-sensitive sodium channels in Drosophila melanogaster. Nature 308: 189-191.

Jackson, F. R., J. Gitschier, G. R. Strichartz, and L. M. Hall (1985) Genetic modifications of voltage-sensitive sodium channels in Drosophila: Gene dosage studies for the seizure locus. J. Neurosci. 5: 1144-1151.

Jackson, F. R., S. D. Wilson, and L. M. Hall (1986) The tip-E mutation of Drosophila decreases saxitoxin binding and interacts with other mutations affecting nerve membranc excitability. J. Ncurogenct. 3 : 19-31.

Jan, Y.-N., and L. Y. Jan (1982) Antibodies to horseradish peroxidase as specific neuronal markers in Drosophila and in grasshopper embryos. Proc. Natl. Acad. Sci. USA 79: 2700-2704. 
Kasbekar, D. P., J. C. Nelson, and L. M. Hall (1987) Enhancer of seizure: A new genetic locus in Drosophila melanogaster defined by interactions with temperature-sensitive paralytic mutations. Genetics 116: 423-431.

Kauvar, L. M. (1982) Reduced $\left({ }^{3} \mathrm{H}\right)$-tetrodotoxin binding in the napis paralytic mutant of Drosophila. Mol. Gen. Genet. 187: 172-173.

Krafte, D. S., T. P. Snutch, J. P. Leonard, N. Davidson, and H. A. Lester (1987) More than one RNA species is involved in controlling inactivation of rat brain sodium channels expressed in Xenopus oocytes. Soc. Neurosci. Abstr. 13: 91.

Kulkarni, S., and A. Padhye (1982) Temperature-sensitive paralytic mutations on the second and third chromosomes of Drosophila melanogaster. Genetic Res. 40: 191-199.

Nelson, I. C., and D. H. Baird (1985) Action potentials persist at restrictive temperatures in temperature sensitive paralytic mutants of adult Drosophila. Soc. Neurosci. Abstr. 12: 313.

Noda, M., T. Ikeda, T. Kayano, H. Suzuki, H. Takeshima, M. Kurasaki, H. Takahashi, and S. Numa (1986) Existence of distinct sodium channel messenger RNA's in rat brain. Nature 320: 188-192.

O'Dowd, D. K., S. Germeraad, and R. W. Aldrich (1987) Expression of sodium currents in embryonic Drosophila neurons: Differential reduction by alleles of the para locus. Soc. Neurosci. Abstr. 13: 577.

Salkoff, L. B., and M. A. Tanouye (1986) Genetics of ion channels. Physiol. Rev. 66: 301-329.

Salkoff, L., A. Butler, M. Hiken, A. Wei, N. Scavarda, K. Griffen, C. Ifune, R. Goodman, and G. Mandel (1987) Genomic organization and deduced amino acid sequence of a putative sodium channel gene in Drosophila. Science 237: 744-749.
Salvaterra, P. M., N. Bournias-Vardiabasis, T. Nair, G. Hou, and C. Lieu (1987) In vitro neuronal differentiation of Drosophila embryo cells. J. Neurosci. 7: 10-22.

Seecof, R. L. (1979) Preparation of cell cultures from Drosophila melanogaster embryos. TCA Manual 5: 1019-1022.

Seecof, R., and R. Unanue (1968) Differentiation of embryonic Drosophila cells in vitro. Exp. Cell Res. 50: 654-660.

Seecof, R., N. Alleaume, L. Teplitz, and I. Gerson (1971) Differentiation of neurons and myoctes in cell cultures made from Drosophila gastrulae. Exp. Cell. Res. 69: 161-173.

Stuhmer, W., C. Methfessel, B. Sakmann, M. Noda, and S. Numa (1987) Patch clamp characterization of sodium channels expressed from rat brain cDNA. Eur. Biophys. J. 14: 131-138.

Tanouye, M. A., C. A. Kamb, L. E. Iverson, and L. Salkoff (1986) Genetics and molecular biology of ionic channels in Drosophila. Annu. Rev. Neurosci. 9: 255-276.

Weiss, R. E., and R. Horn (1986) Functional differences between two classes of sodium channels in developing rat skeletal muscle. Science 233: $361-364$

Wu, C.-F., and B. Ganetzky (1980) Genetic alterations of nerve membrane excitability in temperature-sensitive paralytic mutants of Drosophila melanogaster. Nature 286: 814-816.

Wu, C.-F., B. Ganetzky, L. Y. Jan, Y.-N. Jan, and S. Benzer (1978) A Drosophila mutant with a temperature-sensitive block in nerve conduction. Proc. Natl. Acad. Sci. USA 75: 4047-4057. 